This item was submitted to Loughborough's Research Repository by the author.

Items in Figshare are protected by copyright, with all rights reserved, unless otherwise indicated.

\title{
Brief Report: Training load, salivary immunoglobulin A and illness incidence in elite paratriathletes
}

PLEASE CITE THE PUBLISHED VERSION

https://doi.org/10.1123/ijspp.2018-0439

PUBLISHER

Human Kinetics

VERSION

AM (Accepted Manuscript)

\section{PUBLISHER STATEMENT}

This work is made available according to the conditions of the Creative Commons Attribution-NonCommercialNoDerivatives 4.0 International (CC BY-NC-ND 4.0) licence. Full details of this licence are available at: https://creativecommons.org/licenses/by-nc-nd/4.0/

\section{LICENCE}

CC BY-NC-ND 4.0

\section{REPOSITORY RECORD}

Stephenson, Ben T., Eleanor Hynes, Christof Leicht, Keith Tolfrey, and Vicky Goosey-Tolfrey. 2019. "Brief Report: Training Load, Salivary Immunoglobulin A and Illness Incidence in Elite Paratriathletes". figshare. https://hdl.handle.net/2134/34846. 


\section{Brief Report: Training load, salivary immunoglobulin $A$ and}

2 illness incidence in elite paratriathletes

\section{Original Investigation}

4 Ben T. Stephenson ${ }^{1}$, Eleanor Hynes ${ }^{2}$, Christof A. Leicht ${ }^{1}$, Keith Tolfrey ${ }^{1}$ and Victoria L. 5 Goosey-Tolfrey ${ }^{1}$

6

${ }^{1}$ School of Sport, Exercise \& Health Sciences, The Peter Harrison Centre for Disability Sport, Loughborough University, Loughborough, LE11 3TU, U.K.

${ }^{2}$ School of Sport \& Exercise Sciences, University of Kent, Canterbury, Kent, CT2 7NZ, U.K.

\section{Corresponding author:}

Prof Victoria Goosey-Tolfrey

School of Sport, Exercise \& Health Sciences,

The Peter Harrison Centre for Disability Sport

Loughborough University,

Loughborough,

LE11 3TU,

U.K.

Email: v.l.tolfrey@lboro.ac.uk

Phone +44 (0) 1509226386

Running head: Training load, IgA \& paratriathlon

24 Abstract word count: 249

25 Text-only word count: 1499

26 Figures: 1

Tables: 2 


\section{Brief report: Training load, salivary immunoglobulin A and illness} incidence in elite paratriathletes

Abstract

Purpose: To gain an exploratory insight into the relationship between training load (TL), salivary secretory immunoglobulin A (sIgA) and upper respiratory tract illness (URI) in elite paratriathletes.

Methods: Seven paratriathletes were recruited. Athletes provided weekly saliva samples for the measurement of sIgA over 23 consecutive weeks (February - July) and a further 11 consecutive weeks (November - January). sIgA was compared to individuals' weekly training duration, external TL and internal TL, utilising time spent in pre-determined heart rate zones. Correlations were assessed via regression analyses. URI was quantified via weekly self-report symptom questionnaire.

Results: There was a significant negative relationship between athletes' individual weekly training duration and $\operatorname{sIgA}$ secretion rate $(p=0.028)$ with changes in training duration accounting for $12.7 \%$ of the variance (quartiles: $0.2 \%, 19.2 \%$ ). There was, however, no significant relationship between external or internal TL and sIgA parameters ( $p \geq 0.104)$. There was no significant difference in SIgA when URI was present or not (101\% vs $118 \%$ healthy median concentration; $p \geq 0.225$ ); likewise, there was no difference in sIgA when URI occurred within two weeks of sampling or not ( $83 \%$ vs $125 \%$ healthy median concentration; $p \geq 0.120$ ).

Conclusions: Paratriathletes' weekly training duration significantly affects sIgA secretion rate, yet we did not find a relationship between external or internal TL and SIgA parameters. Further, it was not possible to detect any link between sIgA and URI occurrence which throws into question the potential of using $\operatorname{sig} \mathrm{A}$ as a monitoring tool for early detection of illness.

54 Key words: mucosal immune function, disability, triathlon, monitoring, TRIMP 


\section{Introduction}

Paratriathlon is a variant of triathlon modified for individuals with a physical impairment. ${ }^{1}$ It has been shown that paratriathletes produce large training loads (TLs) to maximise beneficial adaptations. ${ }^{1}$ However, there is a risk high TLs will increase the likelihood of illness, ${ }^{2}$ most commonly upper respiratory tract illness (URI) ${ }^{3}$ which can directly impair performance or limit training availability. ${ }^{2}$

A key antibody in host defence is salivary secretory immunoglobulin A (sIgA). sIgA has been acknowledged as the first line of defence in mucosal immunity. ${ }^{4}$ Several authors have shown that, over prolonged periods, depressions in sIgA, proposed to be modulated by high TLs with insufficient recovery, ${ }^{5}$ increase the likelihood of URI. ${ }^{6,7}$ To date, this research has focused on able-bodied athletes. However, some have studied Paralympic populations, whom may be at heightened risk of URI due to their propensity for excessive overload, and therefore TLs, caused by movement inefficiencies. ${ }^{8}$ Whilst Leicht et al. ${ }^{9}$ presented a negative correlation between TL and SIgA in Paralympic athletes, there is currently little consensus regarding the effects of TL on mucosal immunity in this population. ${ }^{10}$ Furthermore, there has been a wide variety of methods of external (ETL) or internal (ITL) TL representation in the literature, which likely have differing degrees of association to mucosal immunity.

Therefore, the aim of this present study was to gain an exploratory insight into the effects of TL, quantified using objective measures, on sIgA and resultant URI incidence in paratriathletes.

\section{Methods}

\section{Participants}

Seven elite, mixed impairment, paratriathletes participated in this study (Table 1). All provided written informed consent and the procedures were approved by the University Ethical Advisory Committee. All had regularly competed at international level for 2 to 7 years, with six competing at the 2016 Paralympic Games and all reported being free from illness prior to the commencement of the study. Athletes' typical weekly training volume was $11.2 \pm 3.9 \mathrm{~h}$.

\section{Study design}

Saliva samples were collected over 23 consecutive weeks (February - July) and a further 11 consecutive weeks (November - January) whilst athletes undertook their normal training and competition regimes. Athletes visited the laboratory three times (February, July and November) for testing of parameters used in TL quantification. ${ }^{1}$ Due to the variable nature of athletes' schedules it was not possible to collect samples from every athlete each week (11 to 31 samples per athlete).

\section{Saliva collection and analysis}

Samples were collected on the same weekday (06:00 - 08:00 h) every week, before training, 10 minutes after last fluid intake, whilst fasted and before brushing their teeth. Athletes provided a timed, unstimulated saliva sample. Sample volume was estimated assuming a saliva density of $1.00 \mathrm{~g} \cdot \mathrm{ml}^{-1} \cdot{ }^{11}$ Saliva flow rate was calculated from sample volume and collection time. Upon sample provision, athletes completed a questionnaire capturing illness symptoms (14 in total) for the preceding seven days. Athletes recorded the number of days they experienced each symptom and the severity of each symptom on a three-point scale. The number of days each symptom persisted was multiplied by the severity rating and summed to provide an overall quantitative symptom score; a score $\geq 12$ indicated the presence of URI. ${ }^{11}$ Athletes also reported if training availability was affected. IgA concentration was determined by ELISA. ${ }^{9}$ Individuals' healthy median sIgA concentration was calculated as the median of 
concentrations when URI was not present. ${ }^{7}$ Secretion rate (SR) was calculated from $\operatorname{sIgA}$ concentration and saliva flow rate. ${ }^{11}$

\section{Laboratory testing and training load quantification}

Athletes performed both a cycling/handcycling and running/racing-wheelchair incremental exercise test for the determination of heart rate (HR) associated with aerobic (AeLT) and anaerobic (AnLT) lactate thresholds. ${ }^{1}$

Training was represented as total weekly duration for swim, cycle and run training. Resistance training was not included due to its small contribution to total weekly training. Further, ETL accounting for differences in the relative stress of triathlon modalities was calculated (Equation 1). ${ }^{12}$ ITL was calculated from an adaptation of the methods of CejuelaAnta and Esteve-Lanao ${ }^{12}$, incorporating the time spent in pre-determined zones (Equation 2), based on the HR associated with lactate thresholds. ${ }^{1}$ Due to the inability to record HR during swim training, this was represented solely by swim duration. Training duration, ETL and ITL were relativised to the highest individual weekly value recorded during the study period.

\section{Equation 1}

$$
E T L=0.75(\text { swim duration })+0.5(\text { cycling duration })+(\text { run duration })
$$

\section{Equation 2}

$$
\begin{aligned}
& I T L=[0.75(\text { swim duration })]+\left[0.5\left(\text { TIZ1 } 1_{C}+2\left[\text { TIZ2 }_{C}\right]+3\left[\text { TIZ3 }_{C}\right]\right)\right]+\left[\left(T I Z 1_{R}+\right.\right. \\
& \left.\left.2\left[\operatorname{TIZ2}_{R}\right]+3\left[\text { TIZ3 }_{R}\right]\right)\right]
\end{aligned}
$$

TIZ $n_{\mathrm{C}}-$ weekly time (min) spent in zone $n$ during cycling. TIZ $n_{\mathrm{R}}-$ weekly time (min) spent in zone $n$ during running. Zone 1 - below AeLT. Zone 2 - above AeLT, below AnLT. Zone 3 - above AnLT.

\section{Statistical analyses}

Statistical analyses were conducted using SPSS Statistics 23.0 (IBM, New York, USA); statistical significance was set at $p<0.05$. Coefficients of variation were calculated for all saliva variables. Each salivary variable was matched to the athlete's individual TL/training duration for the preceding seven days. A logarithmic transformation was applied to salivary variables to weight increases by a certain factor the same as decreases by the same factor. ${ }^{9}$ Slopes of linear regression lines between salivary variables and TL/training duration were calculated for each athlete and compared, as a group, to a fixed zero with a Wilcoxon statistic. All salivary data were grouped, represented as relative deviation from individuals' healthy median value and compared, via paired-samples t-test or Mann-Whitney U test, to elucidate to the likelihood URI occurrence within two weeks of sample provision. Similarly, salivary variables when URI were present were compared to samples when healthy ( $>2$ weeks to/from URI).

\section{Results}

132 saliva samples were collected. The between- and within-individual variability in $\operatorname{sIgA}$ concentration was $70 \%$ and $40 \%$, respectively, whilst SIgA SR variability was $88 \%$ and $46 \%$, respectively. Athletes' mean $\operatorname{sIgA}$ concentration and SR were $162 \pm 127 \mu \mathrm{g} \cdot \mathrm{ml}^{-1}$ and $78 \pm 76$ $\mu \mathrm{g} \cdot \mathrm{min}^{-1}$, respectively.

There was a significant negative relationship between athletes' total training duration and $\operatorname{sIgA}$ SR $(p=0.028$; Figure 1). The amount of variance in SIgA SR explained by changes in training duration was $12.7 \%$ (quartiles $0.2 \%, 19.2 \%$ ). There was no significant relationship between ETL $(p \geq 0.398)$ or ITL $(p \geq 0.104)$ and SIgA SR or concentration. 
Six athletes reported at least one URI occurrence (range: 0 to 9 URI per athlete) with a total of 22 separate URI episodes. On average, athletes presented with URI every seven weeks and every 8 samples. During 50\% of URI episodes, athletes had to reduce or suspend training. There was no significant difference in relative deviation from individual median $\operatorname{sigA}$ concentration, SR or saliva flow rate between weeks with URI and when healthy $(p \geq 0.228)$ or between samples with URI within two weeks and samples without URI within two weeks $(p \geq 0.120)$ (Table 2).

\section{Discussion}

Training duration explained $12.7 \%$ of the variance in SIgA SR, albeit with large inter-individual variability. The variability is likely due to the individualised nature of which training affected mucosal immunity and we also acknowledge genetic, nutritional and psychological factors that may have an influence. ${ }^{9}$ There was, however, no significant relationship between ETL or ITL and sIgA. Relationships between TL and SIgA have been shown elsewhere in able-bodied sport, $^{7}$ yet, Leicht et al. ${ }^{9}$ are the only researchers to note this interaction in a Paralympic population. This study, however, was conducted in an intermittent ball sport with solely spinal cord injured athletes, thus, was disparate from the multi-impairment endurance sport of paratriathlon. Further, the aforementioned study relied upon subjective measures of TL quantification rather than objective parameters such as HR.

Here, athletes averaged one URI episode every seven weeks, significantly greater than the four URI episodes annually previously reported. ${ }^{6}$ This may be due to Paralympic athletes' vulnerability to illness as a consequence of movement inefficiencies increasing the likelihood of excessive overload. ${ }^{8}$ Nonetheless, in $50 \%$ of URI incidences, athletes stated that their training had been impaired. This highlights the desirability for identifying athletes at risk of illness prior to decrements in training or competitive performance. ${ }^{6}$ The present study, however, noted no relationship between salivary variables and URI incidence. A lack of relationship between sIgA and URI incidence has been reported elsewhere. ${ }^{2,9}$ Although sIgA plays a major role in mucosal immunity, ${ }^{4}$ there are many mechanisms responsible for host defence and insufficiencies in any, not merely $\operatorname{sigA}$, are likely to heighten the risk of illness. ${ }^{7}$

Training duration was the only training measure significantly related to salivary parameters. This may signify a failing of the methods of Cejuela-Anta and Esteve-Lanao ${ }^{12}$ to adequately quantify ETL in its relation to URI incidence. As such, it is likely this method does not truly characterise the stress imposed on the mucosal immune system. Nonetheless, it was surprising that ITL did not relate to $\operatorname{SIgA}$ as this likely better represented the physical stress imposed by training than ETL.

\section{Practical Applications}

This exploratory study found depressions in SIgA SR during periods of high weekly training duration. Due to this, and Paralympic athletes' propensity for illness, we recommend that measures are put in place to minimise the likelihood for missed or impaired training. This includes structuring and accurately monitoring TL to maximise recovery whilst lessening exposure to physical, environmental or psychological stressors. ${ }^{7}$

A limitation to the current study was the lack of mechanistic data to elucidate the relationship between training duration or TL and $\operatorname{sigA}$. Measurement of cortisol, a potential modulating factor in sIgA suppression, ${ }^{2,4}$ may have provided further insight into the causes of variation of $\operatorname{sIg} \mathrm{A}$. 


\section{Conclusions}

190 Paratriathletes' weekly training duration is negatively correlated with sIgA SR, yet there is no 191 significant relationship between ETL or ITL and sIgA parameters. Furthermore, it was not possible to detect any link between sIgA and URI occurrence. 


\section{References}

1. Mujika I, Orbañanos J, \& Salazar H. Physiology and training of a world-champion paratriathlete. Int J Sports Physiol Perform. 2015;10:927-930.

2. Cunniffe B, Griffiths H, Proctor W, Davies B, Baker JS, Jones KP. Mucosal immunity and illness incidence in elite rugby union players across a season. Med Sci Sports Exerc. 2011;4:388-397.

3. Derman W, Schwellnus MP, Jordaan E, et al. Sport, sex and age increase risk of illness at the Rio 2016 Summer Paralympic Games: a prospective cohort study of 51198 athlete days. Br J Sports Med. 2018;52, 17-23.

4. Walsh NP, Gleeson M, Shephard RJ, et al. Position statement part one: Immune function and exercise. Exerc Immunol Rev. 2011;17:6-63.

5. Pyne DB, Gleeson M. Effects of intensive exercise training on immunity in athletes. Int $J$ Sport Med. 1998;19:S183-S194.

6. Gleeson M, McDonald WA, Pyne DB, Cripps AW, Francis JL, Fricker PA, Clancy RL. Salivary IgA levels and infection risk in elite swimmers. Med Sci Sports Exerc. 1999;31:67-73.

7. Neville V, Gleeson M, Folland JP. Salivary IgA as a risk factor for upper respiratory infections in elite professional athletes. Med Sci Sports Exerc. 2008;40, 1228-1236.

8. Blauwet CA, Brook EM, Tenforde AS, et al. Low energy availability, menstrual dysfunction, and low bone mineral density in individuals with a disability: implications for the para athlete population. Sports Med. 2017;47:1697-1708.

9. Leicht CA, Bishop NC, Paulson TAW, Griggs KE, Goosey-Tolfrey V. Salivary immunoglobulin A and upper respiratory symptoms during 5 months of training in elite tetraplegic athletes. Int J Sports Physiol Perform. 2012;7:210-217.

10. Edmonds R, Burkett B, Leicht A, McKean M. Effect of chronic training on heart rate variability, salivary IgA and salivary alpha-amylase in elite swimmers with a disability. PLoS One. 2015;10:e127749.

11. Gleeson M, Bishop N, Oliveira M, McCauley T, Tauler P, Muhamad AS. Respiratory infection risk in athletes: association with antigen-stimulated IL-10 production and salivary IgA secretion. Scand J Med Sci Sports. 2012;22:410-417.

12. Cejuela-Anta R, Esteve-Lanao J. Training load quantification in triathlon. J Human Sport Exerc. 2011;6:1-15. 


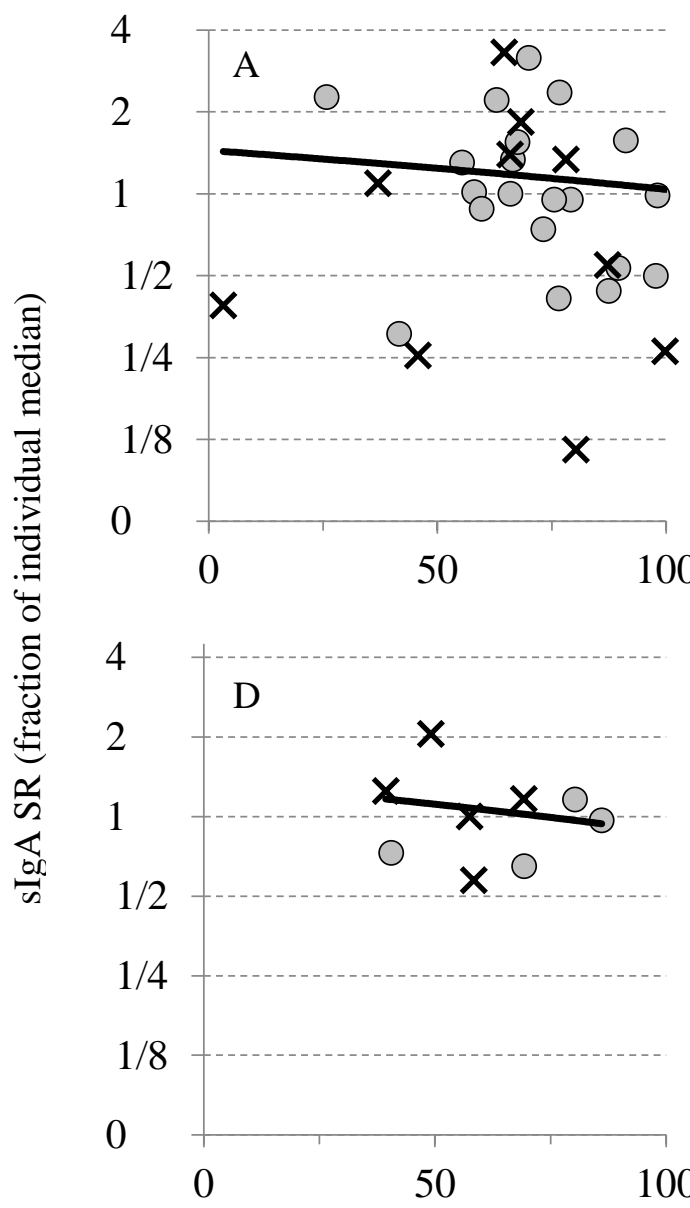

B

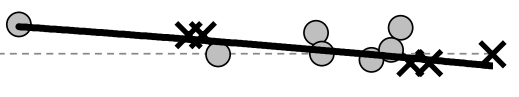

O

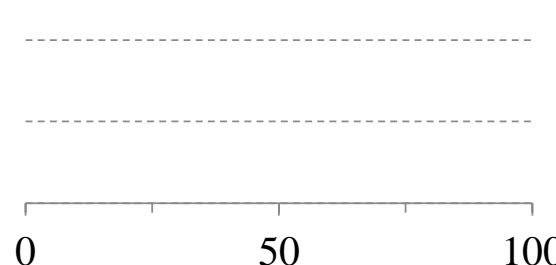

100

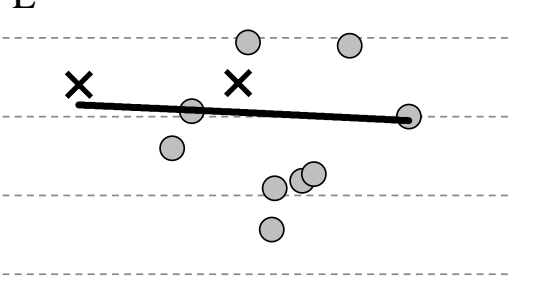

F

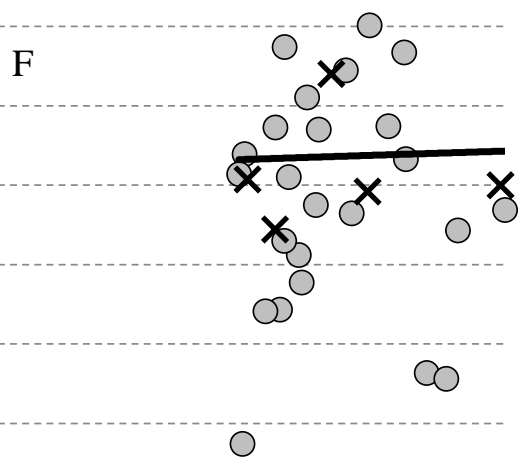

1000

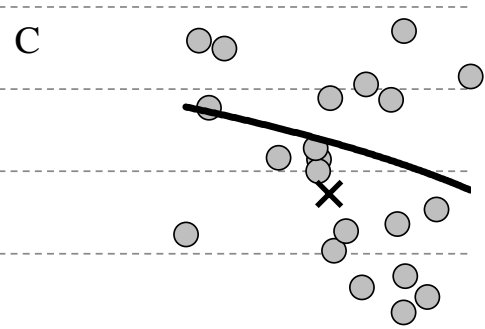

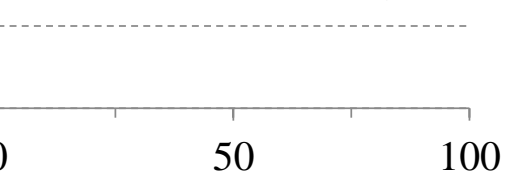
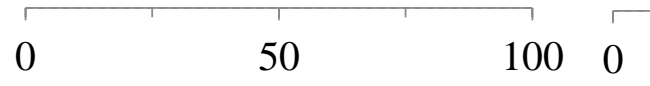

50

G

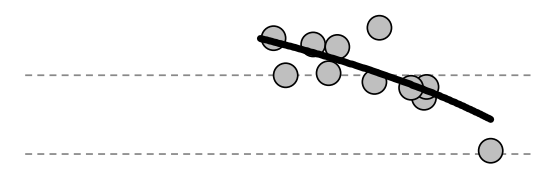

Individual training duration (\%)

Figure 1 Individual athletes' salivary secretory immunoglobulin A (sIgA) secretion rate (SR) plotted against individual training duration with regression lines (lines are distorted by logarithmic scale). $\times$ Samples when URI was present. A-G are participant codes. 
Table 1 Participant characteristics. Values mean \pm standard deviation, where appropriate.

\begin{tabular}{ll}
\hline Age $(\mathrm{y})$ & $30 \pm 10$ \\
Body mass $(\mathrm{kg})$ & $69.5 \pm 6.5$ \\
Cycling $\dot{V} \mathrm{O}_{2 \text { peak }}\left(1 \cdot \mathrm{min}^{-1}\right)$ & $4.06 \pm 0.61$ \\
Sex & 6 male, 1 female \\
Impairment & $1 \mathrm{SCI} \quad(\mathrm{T} 6 \mathrm{i}), 1$ unilateral transfemoral \\
& amputation, 1 hemiplegia cerebral palsy, 3 \\
& unilateral transradial amputation, 1 lower leg \\
& impairment \\
\hline
\end{tabular}

$\overline{\dot{V} \mathrm{O}_{2 \text { peak }} \text { - Peak rate of oxygen uptake. SCI - Spinal cord injury. T6i - Incomplete lesion at the }}$ $6^{\text {th }}$ thoracic vertebra. 
Table 2 Relationship between URI state and URI occurrence within two weeks of sampling date on individual deviation of saliva data, median (quartiles).

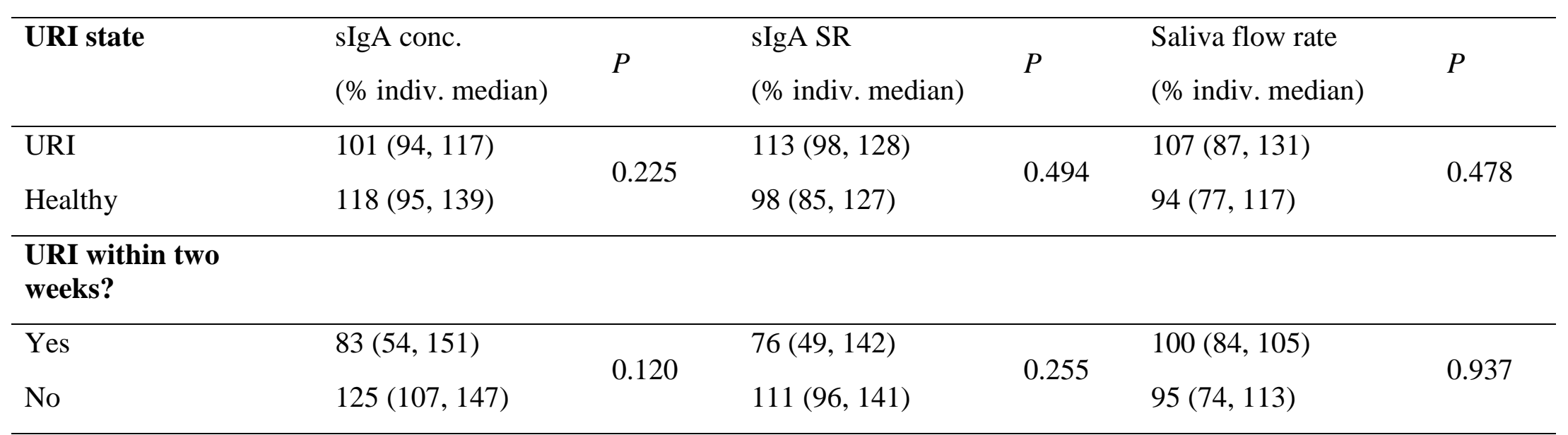

URI - Upper respiratory tract illness. sIgA - Salivary secretory immunoglobulin A. SR - Secretion rate. 